\title{
A SPATIALLY RESOLVED PHOTODISSOCIATION REGION IN THE PLANETARY NEBULA NGC 7027
}

\author{
JAMES R. GRAHAM ${ }^{1}$ \\ Astronomy Department, University of California, Berkeley, California 94720 and Palomar Observatory, California Institute of \\ Technology, Pasadena, California 91125 \\ E. SERABYN \\ Caltech 320-47, Pasadena, California 91125 \\ T. M. HERBST \\ Cornell University, Ithaca, New York 14853 and Max-Plank Institute für Astronomie, Königstuhl 17, D-6900 Heidelberg 1, \\ Germany \\ K. Matthews, G. Neugebauer, AND B. T. Solfer \\ Palomar Observatory, California Institute of Technology, Pasadena, California 91125 \\ T. D. WILSON \\ Palomar Observatory, California Institute of Technology, Pasadena, California 91125 and Department of Physics and \\ Astronomy, University of Rochester, Rochester, New York 14627-0011

\section{S. BECKWITH} \\ Cornell University, Ithaca, New York 14853 and Max-Plank Institute für Astronomie, Königstuhl 17, D-6900 Heidelberg 1, \\ Germany \\ Received 23 July 1992; revised 14 September 1992
}

\begin{abstract}
High spatial resolution, narrow band, infrared line images and CO $(1-0) \mathrm{mm}$ interferometer data are presented for NGC 7027. These data trace emission from the central $\mathrm{H}$ II region $(\mathrm{Br} \alpha)$, the intermediate photodissociation region $\left[\mathrm{H}_{2} 1-0 \mathrm{~S}(1)\right.$ and $3.3 \mu \mathrm{m}$ dust feature], and the molecular circumstellar envelope [CO (1-0)]. The $\mathrm{H}$ II region lies in a cavity in the CO envelope, and consists of a smooth elliptical shell. A striking change of morphology is seen in the $\mathrm{H}_{2}$ emission and the dust feature. The $\mathrm{H}_{2} 1-0 \mathrm{~S}(1)$ emission is composed of two components: (1) an incomplete elliptical ring of knots which bounds the ionized gas; (2) a remarkable thin shell which loops around the $\mathrm{H}$ II region with fourfold symmetry. The dust emission is similar to that from the ionized gas, but is displaced further from the center, and extends at low surface brightness into four "ears" which fill in the bays delineated by the outermost loops of $\mathrm{H}_{2}$ emission. No $3.3 \mu \mathrm{m}$ emission is detectable beyond the outer $\mathrm{H}_{2}$ shell. The outer loops of $\mathrm{H}_{2}$ emission and the $3.3 \mu \mathrm{m}$ emission occupy the region between the edge of the $\mathrm{H}$ II region and the inner edge of the molecular gas. It is natural to ascribe the morphology of NGC 7027 to a photodissociation region which separates the ionized and molecular gas. If this is correct then the exterior $\mathrm{H}_{2}$ loops are due to molecular gas heated by the far-UV emission escaping from the $\mathrm{H}$ II region, and delineate a photodissociation front. The $\mathrm{H}_{2}$ and $\mathrm{CO}$ kinematics rule out shock excitation of the $\mathrm{H}_{2}$ emission and favor UV excitation.
\end{abstract}

\section{INTRODUCTION}

The young planetary nebula NGC 7027 is remarkable on account of its association with a substantial circumstellar molecular envelope (Mufson, et al. 1975). The presence of such a significant envelope, which is probably the remnant of a cool, slow, wind from the progenitor red giant, is unique, and NGC 7027 therefore presents an important opportunity to study the interaction of the newly formed $\mathrm{H}$ II region with the circumstellar material. This interaction takes two forms, namely a mechanical interaction in which a fast wind from the central star ploughs into

${ }^{1}$ Alfred P. Sloan Research Fellow. the slowly moving red giant wind and determines the shape of the resultant planetary nebula (e.g., Kahn \& West 1985; Balick et al. 1987), and a radiative interaction, where farultraviolet radiation $(6 \mathrm{eV}<h v<13.6 \mathrm{eV})$ emerges from the $\mathrm{H}$ II region and dissociates and heats surrounding molecular gas.

This paper describes new high angular resolution infrared line imaging and mm-wave interferometry of NGC 7027 which trace this interaction. The infrared data consist of images of $2.122 \mu \mathrm{m} \mathrm{H}_{2}$ emission, the $3.28 \mu \mathrm{m}$ dust feature and $\mathrm{Br} \alpha$. Emission from $\mathrm{H}_{2}$ was discovered in NGC 7027 by Treffers et al. (1976). Beckwith et al. (1980) demonstrated that the $\mathrm{H}_{2}$ emission was from molecules which are outside the ionized gas. Gatley et al. 


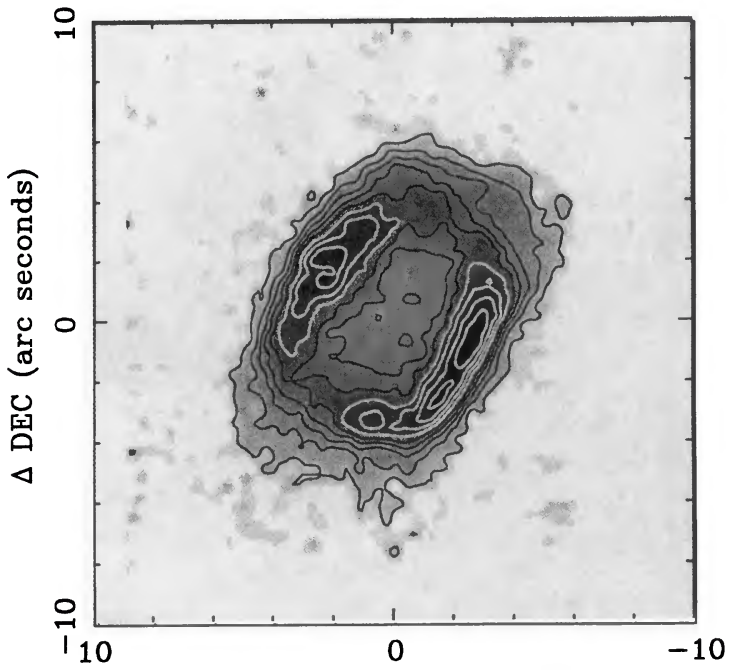

(a)

$\Delta \mathrm{RA}$ (arc seconds)

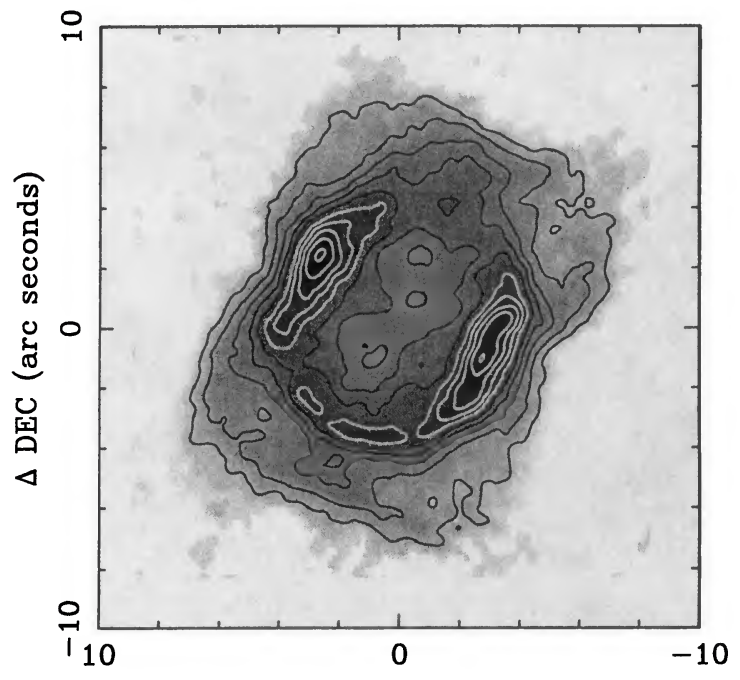

(b)

$\Delta$ RA (arc seconds)

(1988) presented the first $\mathrm{H}_{2}$ line emission image of NGC 7027. Images of the dust feature emission and Br $\alpha$ are presented by Woodward et al. $(1989,1992)$. A preliminary discussion of the current infrared data appears in Graham et al. (1990). The $3.28 \mu \mathrm{m}$ dust feature was discovered by Merrill et al. (1975). Images of this emission have been presented by Woodward et al. (1989, 1992). Interferometer CO $(1-0)$ maps of NGC 7027 have been presented by Masson et al. (1985) and Bieging et al. (1991).

\section{OBSERVATIONS}

Observations of NGC 7027 were made at the Cassegrain focus of the Hale 200 inch telescope with an infrared camera equipped with a Santa Barbara Research Corporation InSb $58 \times 62$ detector array. The pixels project to 0.31 $\times 0$ ". 31 on the sky. The camera is equipped with circular variable filters (CVF) which allow narrow band imaging in the 2 and $3 \mu \mathrm{m}$ windows at $\Delta \lambda / \lambda \simeq 1.3 \%$. Observations in the $2 \mu \mathrm{m}$ window included the lines $\mathrm{H}_{2} 1-0 \mathrm{~S}$ (1) 2.122 $\mu \mathrm{m}$ and continua at 2.09 and $2.19 \mu \mathrm{m}$. The $3 \mu \mathrm{m}$ observa-

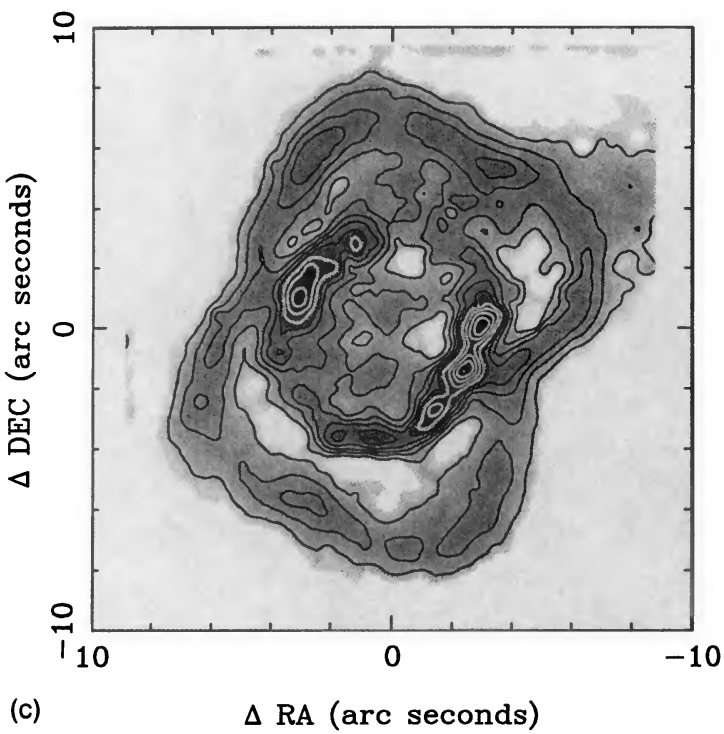

FIG. 1. Continuum subtracted infrared line images presented as contour plots of NGC 7027. All contours start at $10 \%$ of the peak intensity, and increase in linear steps of $10 \%$ up to the peak. A linear grey scale spanning 5\% to the peak is also included. (a) The Br $\alpha$ emission at $4.05 \mu \mathrm{m}$. The first contour is $4.6 \times 10^{-2} \mathrm{erg} \mathrm{s}^{-1} \mathrm{~cm}^{-2} \mathrm{sr}^{-1}$. (b) The $3.28 \mu \mathrm{m}$ dust feature. The first contour is $1.06 \mathrm{erg} \mathrm{s}^{-1} \mathrm{~cm}^{-2} \mathrm{sr}^{-1} \mu \mathrm{m}^{-1}$. (c) The $2.12 \mu \mathrm{m}$ emission. This map traces $\mathrm{H}_{2} 1-0 \mathrm{~S}(1)$. The inner ring of knots, which is coincident with the $\mathrm{H}$ II region also includes a contribution from $\mathrm{He} \mathrm{I} 2.11 \mu \mathrm{m}$. The outer loops are pure $\mathrm{H}_{2}$. The lowest contour is $2.4 \times 10^{-4} \mathrm{erg} \mathrm{s}^{-1} \mathrm{~cm}^{-2} \mathrm{sr}^{-1}$. 


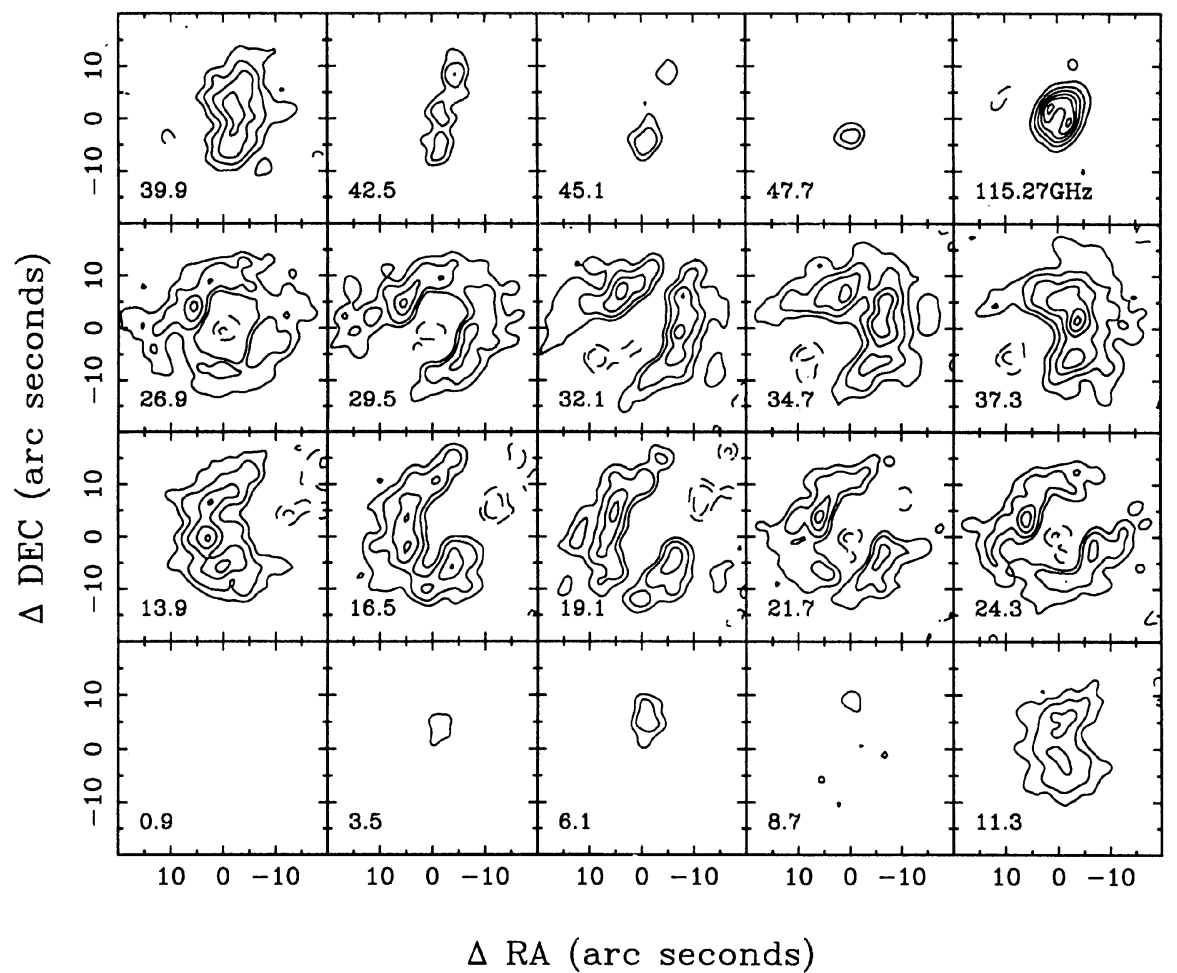

FIG. 2. Interferometer CO $(1-0)$ channel maps of NGC 7027. The maps are continuum subtracted. The number at the lower left of each panel is $v_{\mathrm{LSR}}$ in $\mathrm{km} \mathrm{s}^{-1}$ for that channel. The contour levels are $-0.8,-0.4$, $0.4,0.8,1.6,2.4,3.2$, and $4.0 \mathrm{Jy} /$ beam. Negative contours are dashed. The last panel shows the $115.27 \mathrm{GHz}$ continuum map. The contours are $-0.08,0.08,0.20,0.35,0.50,0.62$, and $0.74 \mathrm{Jy} / \mathrm{beam}$. The synthesized beamwidth is 2"8. The map center is RA 2159.57 DEC 4222.8 (1950.0).

sideband measurements, the continuum flux integrated over the $\mathrm{H}$ II region is $4.6 \mathrm{Jy}$, in good agreement with the earlier measurement of Masson et al. (1985). The resultant continuum subtracted channel maps are shown in Fig. 2.

\section{RESULTS}

\subsection{Infrared Images}

The Br $\alpha$ emission, presented in Fig. 1(a), is composed of a smooth elliptical shell with a minor axis diameter of $6 "$. The intensity is peaked at two symmetrical points along the minor axis, and is very similar to the free-free emission mapped at $\mathrm{cm}$ wavelengths (Masson 1989). At low surface brightness levels ansae extend from the north-west, southeast, and possibly to the south, confirming structure in the $6 \mathrm{~cm}$ map. The current $\mathrm{Br} \alpha$ data are in good agreement with the observations of Woodward et al. (1989).

The dust feature image at $3.28 \mu \mathrm{m}$ [Fig. 1(b)] is, at first sight, rather similar to the emission from the $\mathbf{H}$ II region [Fig. 1(a)]. However, the radius of the prominent central shell is clearly larger than the $\mathrm{H}$ II region (cf. Woodward et al. 1989). Low surface brightness emission extends well beyond the edge of the $\mathrm{H}$ II region. There is some degree ( $\lesssim 5 \%$ ) of limb brightening of this faint emission, particularly at the position adjacent to the southern end of the H II region. The emission has a sharp outer edge with the possible exception of the "blow out" to the north-west where the faint emission extends to the edge of the image.

The $2.12 \mu \mathrm{m}$ morphology is quite remarkable [Fig. 1(c)]. It consists of two components: (1) an incomplete elliptical ring of knots which bounds the ionized gas; (2) a thin shell at a greater radius, which loops around the $\mathrm{H}$ II region with fourfold symmetry at a maximum diameter of $14^{\prime \prime}$. The central ring of knots is almost exactly coincident with the $\mathrm{Br} \alpha$ emission from the $\mathrm{H}$ II region-the east and west limbs are displaced only $\simeq 0$ ". 2 further from the center of the nebula than the Br $\alpha$ emission. The two brightest $2.12 \mu \mathrm{m}$ knots, on the west limb, straddle the Br $\alpha$ peak.

The bandpass of the $2.12 \mu \mathrm{m}$ image was centered on the $\mathbf{H}_{2}$ 1-0 S(1) line. In the $\mathbf{H}$ II region this image includes $\mathrm{H}_{2} 1-0 \mathrm{~S}(1)$ and He I ${ }^{3} P-{ }^{3} S 2.114 \mu \mathrm{m}$. High resolution spectroscopy shows that within the $\mathrm{H}$ II region the $\mathrm{He} I$ line accounts for $20 \%$ of the flux in our bandpass (Smith et al. 1981). Thus, most of the emission at $2.12 \mu \mathrm{m}$ within the $\mathrm{H}$ II region must be due to $\mathrm{H}_{2} 1-0 \mathrm{~S}$ (1). Nonetheless, the $2.12 \mu \mathrm{m}$ CVF image cannot tell whether the central knots are $\mathrm{H}_{2}$ or $\mathrm{He} \mathrm{I}$ emission. This ambiguity is resolved by images of NGC 7027 obtained using the infrared camera in tandem with a Fabry-Perot at $\lambda / \Delta \lambda=3000$ (Herbst \& Beckwith 1988). These Fabry-Perot data resolve the $\mathrm{H}_{2}$ and $\mathrm{He} \mathrm{I}$ lines and show the $\mathrm{H}_{2}$ emission in the $\mathrm{H}$ II region, and reveal that the brightest knots on the east and west 
limbs of the $\mathrm{H}$ II region are indeed knots of $\mathrm{H}_{2}$ emission. Consequently, we infer that $\mathrm{He}$ I $2.114 \mu \mathrm{m}$ is smooth like $\mathrm{Br} \alpha$. This is not surprising because $\mathrm{H} \alpha$ and $\mathrm{He}$ I $5876 \AA$ images of other planetary nebulae are similar (e.g., Aller 1984). The Fabry-Perot observations and unpublished long slit spectroscopy with the grism installed in the infrared camera show that, as expected, the outer loops of emission beyond the $\mathrm{H}$ II region are entirely due to $\mathrm{H}_{2}$ emission. Most of the $\mathrm{H}_{2}$ flux $(\simeq 80 \%)$ comes from these outer loops-in accord with Beckwith et al. (1980) who modeled the $\mathrm{H}_{2}$ emission as a thin shell with a diameter of 12 ".

In Fig. 3 (Plate 7) the three infrared images have been combined into a three-color image to permit comparison of their spatial extend. Figure 3 shows the dust emission extends well beyond the $\mathrm{H}$ II region, and that it is bounded by the $\mathrm{H}_{2}$ emission. The south-east "ear" is clearly filled in with dust emission, while the others are fainter. The overlap of the $\mathrm{H}_{2}$ and dust in the outer regions is particularly interesting. In some places the $\mathrm{H}_{2}$ emission and dust are coextensive (i.e., cyan) all the way to the edge of the nebula, for example to the west near the minor axis, and particularly to the north at the major axis. However, there are other locations where the dust emission is relatively weak compared to the $\mathrm{H}_{2}$ e.g., the south-west loop.

\subsection{Millimeter Maps}

The $\mathrm{CO}(1-0)$ maps in Fig. 2 show a high degree of symmetry in agreement with the results of previous $\mathrm{mm}$ observations (Masson et al. 1985; Bieging et al. 1991). The redshifted, $v_{\mathrm{LSR}}=29.5-40 \mathrm{~km} \mathrm{~s}^{-1}$, and blueshifted material, $v_{\mathrm{LSR}}=11.3-24.3 \mathrm{~km} \mathrm{~s}^{-1}$, form incomplete arcs displaced to the northeast and southwest of the $\mathrm{H}$ II region, respectively. These arcs are shown most clearly in Fig. 4, which displays the emission integrated from $v_{\mathrm{LSR}}=11.3-40$ $\mathrm{km} \mathrm{s}^{-1}$. The integrated emission is dominated by two lobes which lie along the minor axis of the $\mathrm{H}$ II region at a distance of 7" from the center of the nebula. This figure emphasizes the axial symmetry of the $\mathrm{CO}$ distribution.

\section{DISCUSSION}

\subsection{CO Kinematics}

The CO channel maps contain information regarding the kinematics of the molecular envelope of NGC 7027, but this information is rather difficult to extract directly from these maps. Therefore, we present position-velocity diagrams for the major and minor axis of the $\mathrm{CO}$ cloud in Fig. 5. These diagrams are useful because they provide a summary of the kinematic information. The minor axis lies at a position angle (PA) of $65^{\circ}$, passing through the northeast and southwest $\mathrm{CO}$ lobes adjacent to the $\mathrm{H}$ II region. This axis is approximately parallel to the PA of the minor axis of the $\mathrm{H}$ II region ( $60^{\circ}$; Masson 1986).

Figures 5(a) and 5(b) show a ring of emission characteristic of a resolved expanding shell. The most noteworthy aspect of these plots is the large velocity gradients at offsets of $\pm 8^{\prime \prime}$. This abrupt change in velocity leads to a locus of maximum emission which is distinctly box shaped. These figures also emphasize the clumpiness of emission seen in

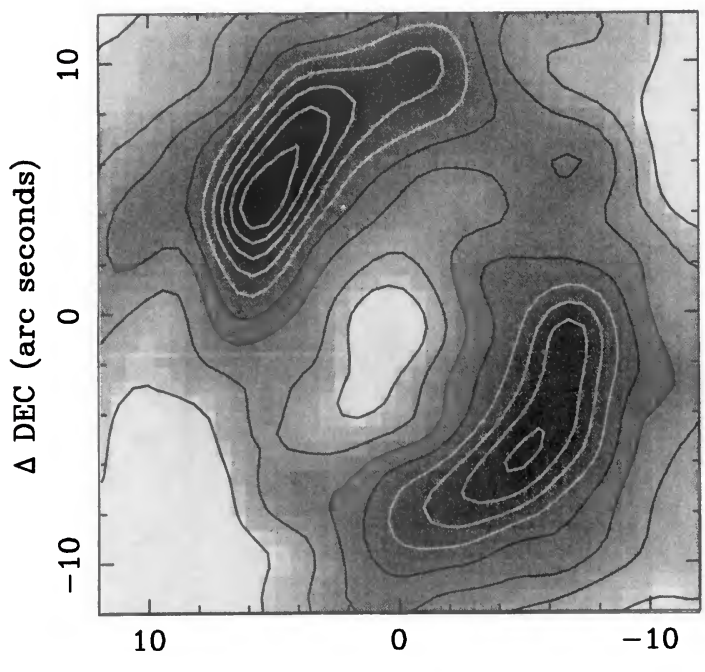

$\Delta \mathrm{RA}$ (arc seconds)

FIG. 4. The integrated $\mathrm{CO}(1-0)$ emission from $v_{\mathrm{LSR}}$ $=11.3-40 \mathrm{~km} \mathrm{~s}^{-1}$ for NGC 7027. The integrated emission consists of two lobes which lie along the minor axis of the $\mathrm{H}$ II region at a distance of 7" from the center of the nebula. Contours run from $10 \%$ of peak in linear intervals of $10 \%$ to the peak. The lowest contour corresponds to an average flux per channel of $0.19 \mathrm{Jy} / \mathrm{beam}$. The synthesized beamwidth is 2 ".8. The data are also displayed as a linear greyscale covering the same data range.

the channel maps and demonstrate that it is also present in the velocity domain, suggesting that the clumps correspond to genuine three-dimensional structures. However, the minor axis plot shows that the two dominant $\mathrm{CO}$ lobes seen in the central velocity channel map are part of an equatorial ring which forms a large, relatively coherent, structure. This is in contrast to the major axis emission distribution which is more fragmented.

Quantitative information regarding the kinematics of NGC 7027 can be found by comparing the position velocity plots with a model consisting of an expanding, optically and geometrically thin, triaxial ellipsoidal shell. This model is similar to those proposed for the $\mathrm{H}$ II region (e.g., Atherton et al. 1979; Masson 1986; Roelfsema et al. 1991). Expansion laws of $v \propto r^{\alpha}$, with $\alpha=-1,0$, and 1 were considered. In all cases the expansion was assumed to be radial. As the model has a large number of free parametersthe axes' lengths, the expansion velocity, $\alpha$, and the inclination-formal least-squares fitting was not performed. Rather, position velocity plots for a large range of parameters were calculated and compared by eye with the observations. The first deduction from this exercise is that only uniformly expanding models $(\alpha=0)$ can account for the "boxy" shape of the locus of maximum emission. Homologous expansion $(\alpha=1)$ predicts ellipsoidal position velocity plots, which are inconsistent with the long straight sections seen in Figs. 5(a) and 5(b). On the other hand $\alpha=-1$ predicts position velocity plots with cusps and concave sections which are not seen.

Uniform radial expansion of a prolate spheroid, inclined to the line of sight, leads to a velocity field which is consistent with the observed position velocity diagrams. For a

\section{(C) American Astronomical Society - Provided by the NASA Astrophysics Data System}



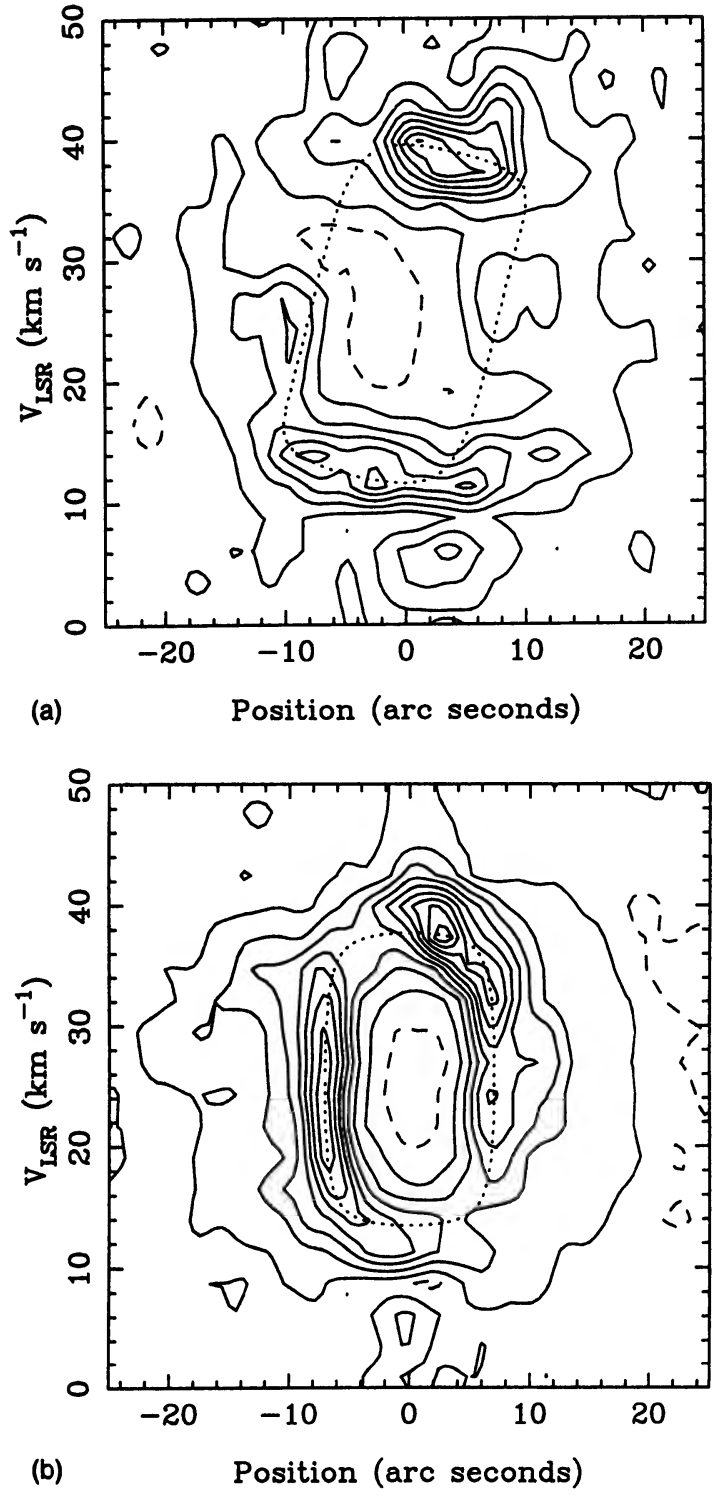

FIG. 5. (a) is the major axis (PA $155^{\circ}$ ) position velocity plot of the CO $(1-0)$ data. (b) is the minor axis (PA 65) position velocity plot. Note the distinctly box-shaped contours which are characteristic of a uniformly expanding prolate ellipsoidal shell. In both plots the dotted line shows the locus of emission from a thin uniformly expanding shell. See Table 1 for the model parameters.

radial flow, the expansion velocity can be determined directly from the separation of the emission peaks along the major axis. The minor axis dimension is the diameter of the ring at the central velocity. The ratio of the extreme velocities in the major and minor axis plots gives the inclination angle. $\mathbf{A}$ uniform expansion model, with parameters which are listed in Table 1, is compared with the observations in Figs. 5(a) and 6(b). It is clear from this comparison that the model provides a good explanation for the kinematic data, and a thin shell can account for a substantial fraction of the observed $\mathrm{CO}$ emission.

Better agreement could undoubtedly be achieved by a model consisting of a thick shell with some velocity dispersion. As the CO $(1-0)$ optical depth probably ap-
TABLE 1. Kinematic model of the CO shell.

\begin{tabular}{ll}
\hline \hline Inclination of major axis to the line of sight & $60^{\circ}$ \\
Major axis radius & $16^{\prime \prime}$ \\
Minor axis radius & $7^{\prime \prime}$ \\
Systemic velocity (LSR) & $25.6 \mathrm{~km} \mathrm{~s}^{-1}$ \\
Expansion velocity & $14 \mathrm{~km} \mathrm{~s}^{-1}$ \\
\hline \hline
\end{tabular}

proaches unity along some lines of sight (Jaminet et al. 1991), a more complete model should also include radiative transfer effects.

\subsection{Comparison of Infrared and $\mathrm{mm}$ Maps}

Figure 6 shows an overlay of the $\mathrm{H}_{2}$ and $\mathrm{CO}$ at the systemic velocity. The correlation between the $\mathrm{H}_{2}$ and $\mathrm{CO}$ emission in the figure is striking. The inner ring and outer loops of $\mathrm{H}_{2}$ sit in the central $\mathrm{CO}$ cavity. Within the limits of the resolution of the $\mathrm{CO}$ data the lowest $\mathrm{CO}$ contour delineates the outer boundary of the $\mathrm{H}_{2}$. The maxima in the $\mathrm{CO}$ along the minor axis are aligned with the indentations in the outer $\mathrm{H}_{2}$ shell along this direction. The secondary pair of $\mathrm{CO}$ peaks, to the north and south, are associated with the pinching in of the $\mathrm{H}_{2}$ shell along the major axis. However there is an offset of $\simeq 35^{\circ}$ between the axes joining the secondary $\mathrm{CO}$ peaks and the $\mathrm{H}_{2}$ indentation along the major axis. The "blowout" seen in faint $\mathrm{H}_{2}$ emission to the north-west lies along the axis with least $\mathrm{CO}$ emission.

Several studies of NGC 7027 show that the CO $(1-0)$ is not very optically thick, i.e., $\tau(1-0) \lesssim 1$, and the intensity is approximately proportional to column density (Deguchi et al. 1990; Jaminet et al. 1991). Thus, CO maps reveal the density structure of the circumstellar molecular gas. If the kinematic model of Sec. 4.1 is correct, then the

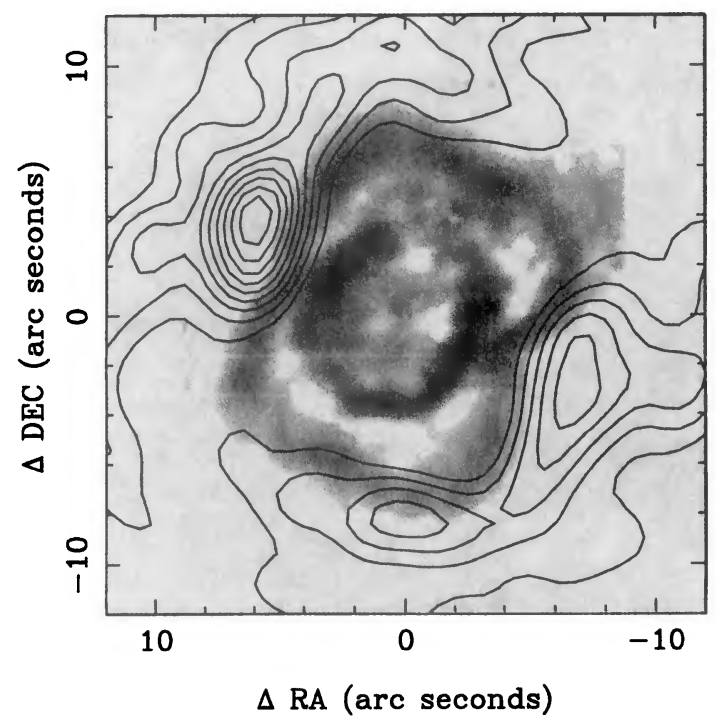

FIG. 6. Contours of $\mathrm{CO}(1-0)$ emission at $v_{\mathrm{LSR}}=25.6$ $\mathrm{km} \mathrm{s}^{-1}$, which corresponds to the central velocity. The contours are linear starting $10 \%$ of peak and increasing in steps of $10 \%$. The lowest contour is $0.5 \mathrm{Jy} / \mathrm{beam}$. This figure also shows the $\mathrm{H}_{2}$ emission displayed as a greyscale. The display corresponds to a linear stretch from $10 \%$ to maximum. 
central channel map corresponds to a planar slice perpendicular to the line of sight through the center of the shell, and this channel should show the envelope in cross section giving the most unambiguous measure of the structure of the molecular envelope free from projection effects.

The current $\mathrm{CO}$ distribution reflects the original spatial distribution of the ejected material and its subsequent modification by the central star. A fast wind from the star forms a bubble of hot, high pressure gas which sweeps up the previous ejecta into a thin shell (e.g., Kahn 1983). If the shock driven by the expanding bubble is fast enough $\left(v_{s} \gtrsim 25-45 \mathrm{~km} \mathrm{~s}^{-1}\right.$; Kwan 1977; Draine et al. 1983) then the swept-up molecular gas is completely dissociated. Ionizing radiation from the star forms an $\mathrm{H}$ II region which expands behind an ionization front. Far-UV radiation (6 $\mathrm{eV}<h v<13.6 \mathrm{eV}$ ) can escape from the $\mathrm{H}$ II region. Since this radiation can dissociate $\mathrm{H}_{2}$, a photodissociation front precedes the ionization front. Shocks and UV light can vibrationally excite $\mathrm{H}_{2}$. Thus the remarkable correlation seen between $\mathrm{CO}$ and $\mathrm{H}_{2}$ in Fig. 6 arises because the outer $\mathrm{H}_{2}$ loops delineate either a shock or a photodissociation front propagating into the molecular envelope. In general, both a shock and a photodissociation region will surround the $\mathrm{H}$ II region. We discuss our observations with the goal of deciding which is responsible for the observed infrared emission.

\subsection{The Origin of the CO Kinematics}

At first sight the kinematic model appears contrived because the ellipsoidal shell at different distances from the central star expands at the same speed. This might suggest that the gas along the poles of the nebula must have decelerated in order to reach a greater distance from the star, yet currently be moving at the same velocity. Implicit in this is the assumption that the $\mathrm{CO}$ which forms the shell was ejected at a single epoch. A much more likely picture is that the $\mathrm{CO}$ originally formed a thick uniformly expanding shell. Interaction with the $\mathrm{H}$ II region then eroded the molecular shell. If the density is lower along one axis, then the cavity will increase in size faster in this direction. The net result is a prolate ellipsoidal shell with the same velocity along the major and minor axes.

\subsection{Shock Excitation}

Several observations suggest supersonic motions are present in the molecular envelope of NGC 7027. Therefore, it is important to investigate the role of shocks in determining the observed morphology at infrared and $\mathrm{mm}$ wavelengths. However, we anticipate the conclusion of this and the following section by stating that shocks are probably not responsible for exciting the $\mathrm{H}_{2}$ emission or its relationship to the $\mathrm{CO}$.

Figure 6 shows that the morphology of the central CO cavity does not match the $\mathrm{H}$ II region, but rather the outer $\mathrm{H}_{2}$ loops. This can be explained if a dissociative shock propagates ahead of the ionization front into the molecular gas. The dereddened average surface brightness of the $\mathrm{H}_{2}$ $1-0 \mathrm{~S}(1)$ emission in the loops is $\simeq 8 \times 10^{-4}$

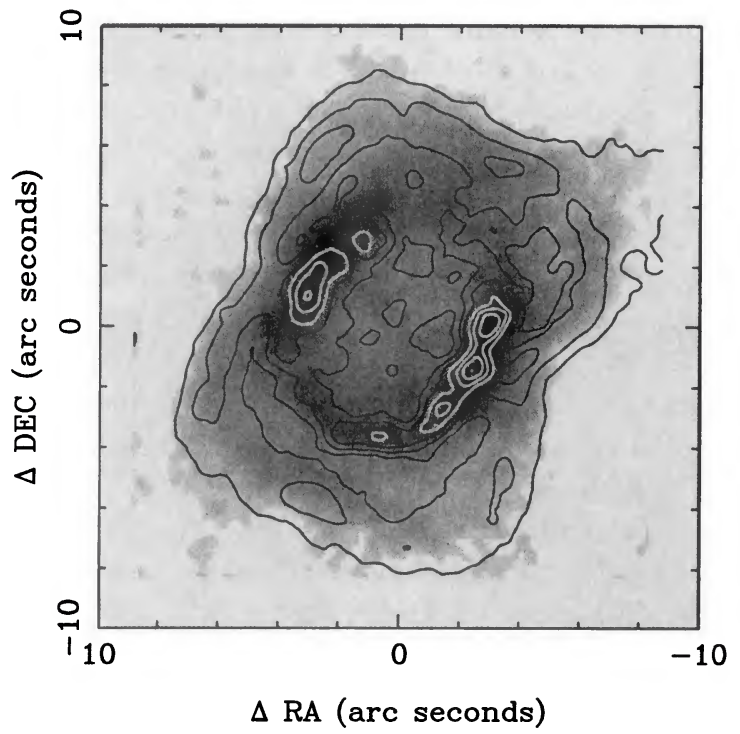

FIG. 7. Comparison of the $3.3 \mu \mathrm{m}$ dust feature, displayed as a greyscale, with contours of $\mathrm{H}_{2}$ emission. The $3.3 \mu \mathrm{m}$ dust emission is bounded by the $\mathbf{H}_{2}$, providing compelling evidence that the outer $\mathrm{H}_{2}$ loops delineate a photodissociation front.

erg s ${ }^{-1} \mathrm{~cm}^{-2} \mathrm{sr}^{-1}$, assuming $A_{K}=0.34 \mathrm{mag}$ (Woodward et al. 1992). For a density in the equatorial plane of $n_{\mathrm{H}_{2}} \simeq 3 \times 10^{4} \mathrm{~cm}^{-3}$ (Jaminet et al. 1991) a shock velocity of $v_{s} \simeq 25 \mathrm{~km} \mathrm{~s}^{-1}$ is required (Draine et al. 1983). There is no observational evidence for such fast shocks in NGC 7027.

The highest velocity $\mathrm{CO}(1-0)$ emission is observed at $v_{\mathrm{LSR}}=3.5$ and $47.7 \mathrm{~km} \mathrm{~s}^{-1}$, i.e., $\Delta v \simeq \pm 22.5 \mathrm{~km} \mathrm{~s}^{-1}$ (cf. Fig. 2). This emission is located close to the edge of the $\mathrm{H}$ II region at its northern and southern ends. Masson et al. (1985) also reported high velocity emission towards the center of the nebula, and attributed it to a layer of shock accelerated neutral gas surrounding the $\mathrm{H}$ II region. The similarity of the expansion speed of the high velocity $\mathrm{CO}$ and the ionized gas $\left(23 \mathrm{~km} \mathrm{~s}^{-1}\right.$, e.g., Roelfsema et al. 1991), suggests that the surrounding molecular gas has been shock accelerated by the expansion of the $\mathrm{H}$ II region (cf. Jaminet 1991). The resulting shock speed is $v_{s} \simeq 8.5$ $\mathrm{km} \mathrm{s}^{-1}$ for the isothermal case, given the observed expansion speeds of the ionized and molecular gas. The principal coolants of slow shocks $\left(v_{s}<15 \mathrm{~km} \mathrm{~s}^{-1}\right)$ in dense molecular gas $\left(n_{\mathrm{H}}=10^{4}-10^{6} \mathrm{~cm}^{-3}\right)$ are pure rotational lines of $\mathrm{H}_{2}$ and [O I] fine structure lines (Draine et al. 1983; McKee et al. 1984). Consequently, the molecular shock in the envelope of NGC 7027 is too slow to produce observable 1-0 S(1) emission. The kinematics of the $\mathrm{H}_{2}$ emission, discussed in Sec. 4.5, confirm this conclusion.

\subsection{A Photodissociation Region Surrounding NGC 7027}

An alternative explanation for the cavity in the $\mathrm{CO}$ shell and the close correlation with the outer $\mathrm{H}_{2}$ loops is that the $\mathrm{H}_{2}$ emission comes from radiatively heated gas in a photodissociation region. The thin, limb brightened $\mathrm{H}_{2}$ morphology is consistent with emission from a photodissociation

\section{(C) American Astronomical Society - Provided by the NASA Astrophysics Data System}


region (Tielens \& Hollenbach 1985). Figure 7 compares the $3.3 \mu \mathrm{m}$ dust feature with the $\mathrm{H}_{2}$ emission and shows that they are coextensive outside the $\mathbf{H}$ II region. (See also Fig. 3.) The $3.3 \mu \mathrm{m}$ dust feature usually delineates the interfaces between ionized and molecular gas (e.g., Sellgren et al. 1990). Therefore, this figure provides compelling evidence for a photodissociation region between the ionized gas and the inner edge of the CO cavity.

Far-UV radiation excites both $\mathrm{H}_{2}$ and $3.3 \mu \mathrm{m}$ dust emission. The excitation of these species occurs in distinct parts of the photodissociation region, and the emission should have a distinctive morphology. The photodissociation front is located at a distance equivalent to $A_{V}=1-2 \mathrm{mag}$ into the neutral cloud, and corresponds to the penetration depth of far-UV light (Tielens \& Hollenbach 1985). The exact position depends upon the relative importance of dust extinction and molecule self shielding. The $\mathrm{H}_{2}$ is collisionally excited in UV heated gas; the abundance of vibrationally excited $\mathrm{H}_{2}$ is sharply peaked at the photodissociation front (Tielens \& Hollenbach 1985; Burton et al. 1990). The 3.3 $\mu \mathrm{m}$ feature is due to the fundamental $\mathrm{C}-\mathrm{H}$ stretch mode of hydrogenated bonds on carbon molecules such as polycyclic aromatic hydrocarbons (e.g., Puget \& Leger 1989). The $3.3 \mu \mathrm{m}$ emission is fluorescently excited by far-UV light and therefore traces the penetration of this radiation into the gas surrounding the $\mathrm{H}$ II region. Figures 3 and 7 show that the $3.3 \mu \mathrm{m}$ dust emission extends well beyond the edge of the $\mathrm{H}$ II region, but that it is bounded by the outer loops of $\mathrm{H}_{2}$ emission. This is precisely the morphology expected for a photodissociation front where the propagation of far-UV light is determined by dust extinction.

The size of the gap between the $\mathrm{H}$ II region and the $\mathrm{CO}$ is correct for a photodissociation region assuming that attenuation of far-UV is dominated by dust and standard extinction- $N_{\mathrm{H}}=1.6 \times 10^{21} A_{V} \mathrm{~cm}^{-2}$. The minor axis dimensions of the $\mathrm{H}$ II region and the $\mathrm{CO}$ shell are 3 " and 7", respectively. Thus, the width of the photodissociation region is $\simeq 4^{\prime \prime}$. At a distance of $880 \mathrm{pc}$ and with $n_{\mathrm{H}_{2}}=3$ $\times 10^{4} \mathrm{~cm}^{-3}$, the column in the photodissociation region is $\simeq 3 \times 10^{21} \mathrm{~cm}^{-2}$. This column of gas corresponds to $A_{V}$ $=1.8 \mathrm{mag}$, and falls within the expected range of 1-2 mag. The larger size of the $\mathrm{CO}$ shell along the major axis presumably indicates a corresponding lower density in this direction.

The observed $\mathrm{H}_{2}$ surface brightness is similar to that expected for the physical conditions in NGC 7027 according to photodissociation region models (cf. Black \& Van Dishoeck 1987; Burton et al. 1990). If the density in the equatorial plane at the edge of the $\mathrm{H}$ II region is $n_{\mathrm{H}_{2}}$ $\simeq 3 \times 10^{4} \mathrm{~cm}^{-3}$ and the far-UV radiation field is $2 \times 10^{3}$ times the interstellar field at a radius of $7 "$ (Black \& van Dishoeck 1987) then $I_{1-0 \text { (1) }}=5.5 \times 10^{-5}$ $\mathrm{erg} \mathrm{s}^{-1} \mathrm{~cm}^{-2} \mathrm{sr}^{-1}$ (Sternberg 1988). Models of photodissociation regions show that the vibrationally excited $\mathrm{H}_{2}$ emission comes from a region $\delta R / R \simeq 0.1$ (Tielens \& Hollenbach 1985). The limb brightening factor for a spherical shell of radius $R$, and thickness $\delta R$, is $2(2 R / \delta R)^{0.5}$. Therefore, the peak $1-0 \mathrm{~S}(1)$ surface brightness excited by far-UV radiation should be $4.9 \times 10^{-4} \mathrm{erg} \mathrm{s}^{-1} \mathrm{~cm}^{-2} \mathrm{sr}^{-1}$, or about $60 \%$ of the typical observed $\mathrm{H}_{2}$ surface brightness. The calculated surface brightness depends on uncertain properties of the circumstellar shell, such as the gas temperature, the gas-to-dust ratio, the optical properties of the dust grains, and the details of the surface chemistry which lead to formation of $\mathbf{H}_{2}$. For example, different grain models lead to fluxes which vary by a factor of about 3 (Black \& Van Dishoeck 1987). Consequently, the measured infrared $\mathrm{H}_{2}$ flux is in accord with that calculated for the photodissociation region model.

CO observations imply that the density is comparable to the critical density $\left(n_{\mathrm{H}_{2}}^{\text {crit }} \simeq 10^{4} \mathrm{~cm}^{-3}\right.$ ) for thermalization of the lower $(v \lesssim 2)$ vibrational levels of $\mathbf{H}_{2}$. Therefore, measurement of the relative intensity of $v=1-0$ and $v=2-1$ transitions cannot be used as an unambiguous diagnostic for UV heating (Sternberg \& Dolgarno 1989). On the other hand, the $\mathrm{H}_{2}$ rotation and vibrational populations in NGC 7027 are not in LTE. This observation lead Tanaka et al. (1989) to decompose the $\mathrm{H}_{2}$ emission into a mixture of thermal and fluorescent components. As Tanaka et al. point out, the thermal component does not necessarily mean shocked gas. The presence of both components is qualitatively consistent with emission from a dense photodissociation region. The detection of [C II] $158 \mu \mathrm{m}$ emission for NGC 7027 is also consistent with the photodissociation region interpretation (Genzel et al. 1988).

Shock excited and photodissociation region $\mathrm{H}_{2}$ emission have distinct kinematic signatures. Emission from a photodissociation region is found at the rest velocity of the illuminated gas, while shock excited emission comes from accelerated gas and therefore is seen at a different velocity from the ambient, unshocked material. For an isothermal shock the velocity difference is equal to the shock velocity (Spitzer 1978). A high resolution spectrum of the $1-0 S(1)$ emission from NGC 7027, in a small beam passing through the center of the nebula, shows a double peaked profile characteristic of an expanding shell (Geballe 1990). The separation of the peaks corresponds to an expansion velocity of $15 \mathrm{~km} \mathrm{~s}^{-1}$. There is none of the high velocity molecular gas ( $\left.\delta v= \pm 40 \mathrm{~km} \mathrm{~s}^{-1}\right)$ which would be present if the $\mathrm{H}_{2}$ emission were shock excited. On the contrary, the $\mathrm{H}_{2}$ shell is expanding at essentially the same speed as the $\mathrm{CO}$ shell. Therefore, the simplest interpretation is that the bulk of the $\mathbf{H}_{2}$ emission comes from unshocked gas which is part of a photodissociation region at the inner edge of the $\mathrm{CO}$ shell.

\subsection{Comparison with the Orion Bar}

Similar stratification of ionized gas, dust feature emission and vibrationally excited $\mathrm{H}_{2}$ is found in the Orion Bar (Sellgren et al. 1990), and is one of the reasons for attributing the $3.3 \mu \mathrm{m}$ and $\mathrm{H}_{2}$ emission to a photodissociation region. The similarity between the Orion photodissociation region and NGC 7027 is also quantitative because the ratio of peak $\mathrm{H}_{2}$ and dust surface brightnesses are also comparable: $I_{\lambda}(3.3 \mu \mathrm{m}) / I_{\mathrm{H}_{2}}=1700 \mu \mathrm{m}^{-1}$ in the Orion photodissociation region; $I_{\lambda}(3.3 \mu \mathrm{m}) / I_{\mathrm{H}_{2}}=2900 \mu \mathrm{m}^{-1}$ for NGC 7027. 
The main difference between the Orion bar and NGC 7027 is that $\mathrm{H}_{2}$ and $3.3 \mu \mathrm{m}$ emitting dust are present in the $\mathrm{H}$ II region in NGC 7027. This difference is most likely due to the youth of the ionized region in NGC 7027 [ $\simeq 600 \mathrm{yr}$, Masson (1989)]. PAHs are destroyed and dehydrogenated inside an $\mathrm{H}$ II region by the ionizing flux and hot electrons, but the time scale must be longer than the age of NGC 7027. If there has been inadequate time for substantial destruction of PAHs in NGC 7027, then the larger value of $I_{\lambda}(3.3 \mu \mathrm{m}) / I_{\mathrm{H}_{2}}$ in NGC 7027 may also be due to the youth of the nebula. The presence of the inner ring of $\mathrm{H}_{2}$ adjacent to the $\mathrm{H}$ II is harder to explain. The implication is that some $\mathrm{H}_{2}$ survives the passage of the photodissociation front. This would point to the presence of dense molecular knots in which $\mathbf{H}_{2}$ can survive the interval between the arrival of the photodissociation and ionization fronts.

\section{CONCLUSIONS}

We have presented high resolution infrared and $\mathrm{mm}$ line data for the planetary nebula NGC 7027 which allow us to investigate the nature of the transition region between the outer edge of the $\mathrm{H}$ II region, and the inner edge of the nebula's molecular envelope. This region is traced by vibrationally excited $\mathrm{H}_{2}$ emission and the $3.3 \mu \mathrm{m}$ feature of interstellar dust. The $\mathrm{H}_{2}$ morphology is particularly striking, and consists of an inner shell of emission adjacent to the $\mathrm{H}$ II region, and outer loops which are coincident with the inner edge of the CO shell.

The emission is stratified, with $\mathrm{Br} \alpha, 3.3 \mu \mathrm{m}$ dust and $\mathrm{H}_{2}$ occupying a central cavity in the $\mathrm{CO}$ distribution. Both the dust feature and the $\mathrm{H}_{2}$ emission extend well beyond the edge of the ionized gas and bridge the gap between the edge of the $\mathrm{H}$ II region and the inner edge of the molecular gas. The $\mathrm{H}_{2}$ bounds the $3.3 \mu \mathrm{m}$ dust emission. This morphology is characteristic of a photodissociation region. The observed $\mathrm{H}_{2}$ surface brightness is consistent with the emission from far-UV heated molecular gas.

The kinematics of the $\mathrm{CO}$ are in accord with emission from a shell expanding uniformly at $14 \mathrm{~km} \mathrm{~s}^{-1}$. Comparison of the CO observations with Geballe's (1990) high resolution spectrum of the $\mathrm{H}_{2} 1-0 \mathrm{~S}$ (1) line show that the bulk of the $\mathrm{H}_{2}$ emission comes from gas at the same velocity as the $\mathrm{CO}$ shell, i.e., unshocked gas. The $\mathrm{CO}$ channel maps show regions of gas shock accelerated by the expansion of the $\mathrm{H}$ II region. However, these shocks are too slow to excite observable $\mathrm{H}_{2}$ emission.

J. R. G. is supported in part by an Alfred P. Sloan Research Fellowship. Infrared astrophysics at Caltech is supported by a grant from the NSF. Observations at Palomar were made as part of a continuing collaborative agreement between Cornell and Caltech. We thank Juan Carrasco, the night assistant at the 200 inch telescope, and the entire staff of Palomar observatory. We acknowledge valuable discussions on photodissociation regions with Xander Tielens.

\section{REFERENCES}

Aller, L. H. 1984, Physics of Thermal Gaseous Nebulae (Reidel, Dordrecht)

Atherton, P. D., Hicks, T. R., Reay, N. K., Robinson, G. J., \& Worswick, S. P. 1979, ApJ, 232, 786

Balick, B., Preston, H. L., \& Icke, V. 1987, AJ, 94, 1641

Beckwith, S., Neugebauer, G., Becklin, E. E., Matthews, K., \& Persson, S. E. 1980, AJ, 85, 886

Bieging, J. H., Wilner, D., \& Thronson, H. A. 1991, ApJ, 379, 271

Black, J. H., \& Van Dishoeck, E. F. 1987, ApJ, 322, 412

Burton, M. G., Hollenbach, D. J., \& Tielens, A. G. G. M. 1990, ApJ, 365, 620

Deguchi, S., Izumiura, H., Kaifu, N., Mao, X., Nguyen-Q.-Rieu, \& Ukita, N. 1990, ApJ, 351, 522

Draine, B. T., Roberge, W. G., \& Dalgarno, A. 1983, ApJ, 264, 485

Elias, J. H., Frogel, J. A., Matthews, K., \& Neugebauer, G. 1982, AJ, 87, 1031

Gatley, I. Depoy, D. L. \& Fowler, A. M. 1988, Science, 242, 1264

Graham, J. R., Matthews, K., Neugebauer, G., Soifer, B. T., Wilson, T

D., Beckwith, S., \& Herbst, T. 1990, BAAS, 22, 813

Geballe, T. R. 1990, in Molecular Astrophysics, edited by T. W.

Hartquist (Cambridge University Press, Cambridge), p. 345

Genzel, R., Harris, A. I., \& Stutzki, J. 1988, Infrared Spectroscopy in Astronomy, Proceedings of the 22nd ESLAB Symposium, edited by B.

H. Kaldeich (ESTEC, Noordwijk, The Netherlands), p. 115

Herbst, T. M., \& Beckwith, S. 1988, PASP, 100, 635

Hollenbach, D. J., Takahashi, T., \& Tielens, A. G. G. M. 1991, ApJ, 377, 192

Jaminet, P. A., Danchi, W. C., Sutton, E. C., Russell, A. P. G.,Sandell,

G., Biejing, J. H., \& Wilner, D. 1991, ApJ, 380, 461

Kahn, F. D. 1983, in Planetary Nebulae, IAU Symposium No. 103, ed- ited by D. R. Flower (Reidel, Dordrecht), p. 305

Kahn, F. D., \& West, K. A. 1985, MNRAS, 212, 837

Kwan, J. 1977, ApJ, 216, 713

Masson, C. R., et al. 1985, ApJ, 292, 464

Masson, C. R. 1986, ApJ, 302, L27

Masson, C. R. 1989, ApJ, 336, 294

McKee, C. F., Chernoff, D. F., \& Hollenbach, D. J. 1984, in Galactic and ExtraGalactic Infrared Spectroscopy, edited by M. F. Kessler and J. P. Phillps (Reidel, Dordrecht), p. 103

Merrill, K. M., Soifer, B. T., \& Russell, R. W. 1975, ApJ, 200, L37

Mufson, S. L., Lyon, J., \& Marionni, P. A. 1975, ApJ, 201, L85

Oke, J. B., \& Shild, R. E. 1970, ApJ, 161, 1015

Puget, J. L., \& Leger, A. 1989, ARA\&A, 27, 161

Roelfsema, P. R., Goss, W. M., Pottasch, S. R., \& Zijlstra, A. 1991, A\&A, 251, 611

Sellgren, K., Tokunaga, A. T., \& Nakada, Y. 1990, ApJ, 349, 120

Smith, H. A., Larson, H. P., \& Fink, U. 1981, ApJ, 244, 835

Spitzer, L. 1978, in Physical Processes in the Interstellar Medium (Wiley, New York), p. 218

Sternberg, A. 1988, ApJ, 332, 400

Sternberg, A., \& Dalgarno, A. 1989, ApJ, 338, 197

Tanaka, M., Hasegawa, T., Hayashi, S. S., Brand, P. W. J. L., \& Gatley, I. 1989, ApJ, 336, 207

Tielens, A. G. G. M., \& Hollenbach, D. 1985, ApJ, 291, 722

Treffers, R. R., Fink, V., Larson, H. P., \& Gautier, T. N. 1976, ApJ, 209, 793

Woodward, C. E., Pipher, J. L., Shure, M., Forrest, W. J., \& Sellgren, K. 1989, ApJ, 342, 860

Woodward, C. E., Pipher, J. L., Forrest, W. J., Moneti, A., \& Shure, M. A. 1992, ApJ, 385, 567 


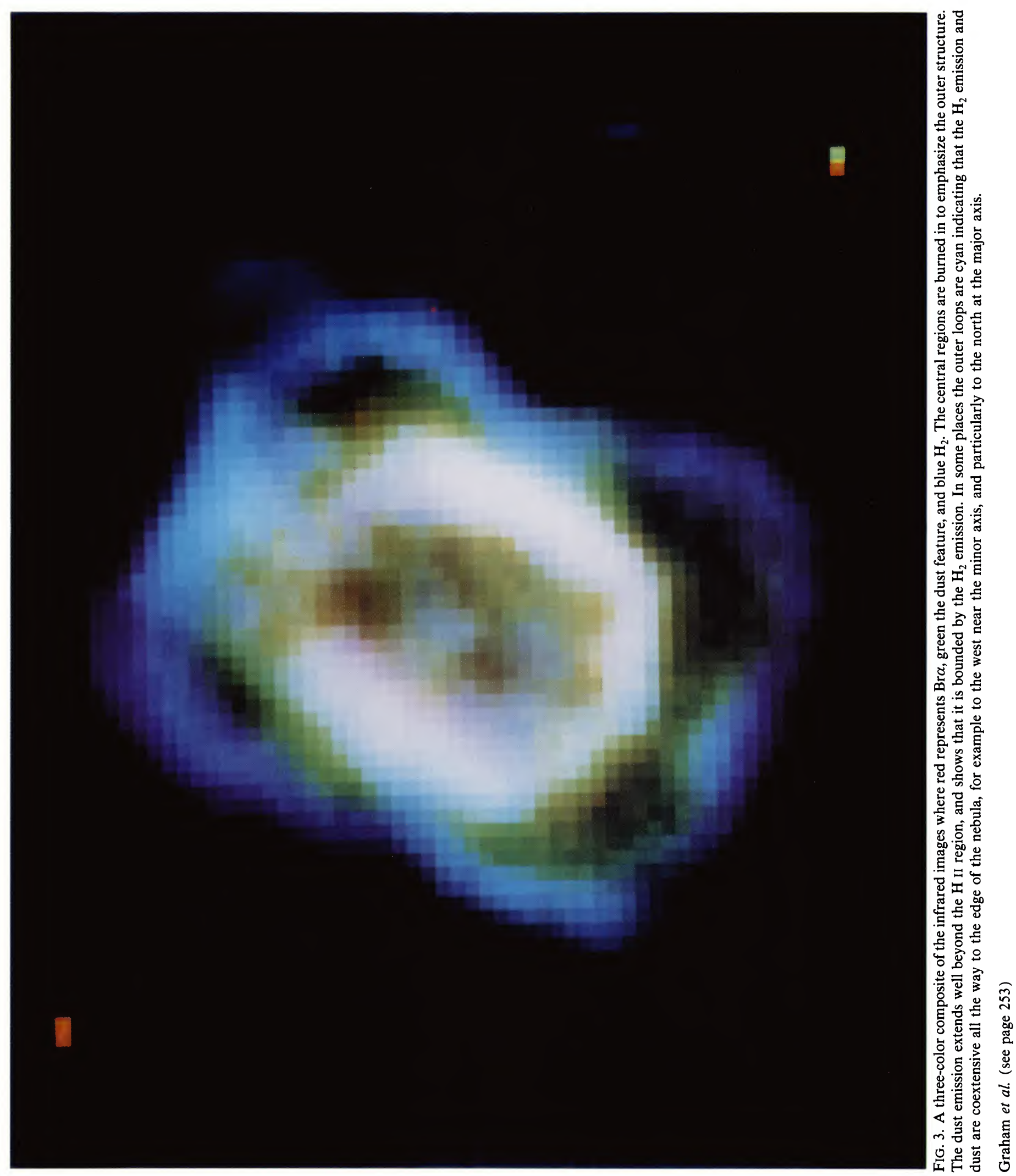




\section{THE ASTRONOMICAL}

\section{JOURNAL}

FoundED BY B. A. Gould

1849
UNINITY COLLEG LIBRALA RECEIVED

JAN 131993

Published for the

American Astronomical Society by the 